\title{
Bleeding and thromboembolism due to drug-drug interactions with non-vitamin K antagonist oral anticoagulants-a Swedish, register-based cohort study in atrial fibrillation outpatients
}

\author{
Johan Holm $^{1,2}$ (D) - Buster Mannheimer ${ }^{3,4}$ (D) Rickard E Malmström $^{2,5} \cdot$ Erik Eliasson $^{1,2}$ (D) Jonatan D Lindh $^{1,2}$ (D)
}

Received: 26 August 2020 / Accepted: 1 October 2020 / Published online: 7 October 2020

(C) The Author(s) 2020

\begin{abstract}
Purpose To study the association between interacting drugs and bleeding or thromboembolism in atrial fibrillation outpatients treated with non-vitamin K antagonist oral anticoagulants (NOACs).

Methods Population-based cohort study of outpatients treated with NOACs in Sweden from 2008 to 2017. Patients with atrial fibrillation and newly initiated NOAC treatment were identified in the Prescribed Drug Register. Comorbidities and outcome data were retrieved from the Patient Register and the Cause of Death Register. Cox-regression analyses were performed to evaluate the primary endpoints any severe bleed and ischemic stroke/transient ischemic attack/stroke unspecified during the first six months of treatment. Secondary endpoints were gastrointestinal bleeding, intracranial bleeding, ischemic stroke, and venous thromboembolism.

Results Increased risk of any severe bleed was found when NOAC treatment, and drugs with pharmacodynamic effect on bleeding were combined, compared to NOAC only. An increased risk with these combinations was evident for apixaban (hazard ratio (HR) 1.47; 95\% CI 1.33-1.63), rivaroxaban (HR 1.7; 95\% CI 1.49-1.92), and dabigatran (HR 1.26; 95\% CI 1.05-1.52). For apixaban, there was an increased risk of any severe bleed when combined with CYP3A4 and/or P-glycoprotein (P-gp) inhibitors (HR 1.23; 95\% CI 1.01-1.5). The use of inducers of CYP3A4 and/or P-gp was low in this cohort, and effects on ischemic stroke/ TIA/stroke unspecified could not be established.

Conclusion Increased risk of bleeding was seen for pharmacodynamic and pharmacokinetic interactions with NOACs. Prescribers need to be vigilant of the effect of interacting drugs on the risk profile of patients treated with NOACs.
\end{abstract}

Keywords Anticoagulants $\cdot$ Drug interactions $\cdot$ Hemostasis $\cdot$ Pharmacokinetics $\cdot$ Thrombosis

Erik Eliasson and Jonatan D. Lindh should be considered joint senior author

Electronic supplementary material The online version of this article (https://doi.org/10.1007/s00228-020-03015-7) contains supplementary material, which is available to authorized users.

Johan Holm

johan.holm@ki.se

1 Division of Clinical Pharmacology, Department of Laboratory Medicine, Karolinska Institutet, Stockholm, Sweden

2 Clinical Pharmacology, Karolinska University Hospital, Stockholm, Sweden

3 Department of Clinical Science and Education at Södersjukhuset, Karolinska Institutet, Stockholm, Sweden

4 Internal Medicine, Södersjukhuset, Stockholm, Sweden

5 Department of Medicine Solna, Karolinska Institutet, Stockholm, Sweden

\section{Introduction}

Non-vitamin K antagonist oral anticoagulants (NOACs) have become increasingly utilized in Sweden and the rest of the world during the past two decades, and the drug group is the first-line treatment for stroke prevention in atrial fibrillation [1-4]. Apixaban is more commonly chosen than the other NOACs, whereas rivaroxaban, dabigatran, and edoxaban, in descending order of use, are also increasingly used among Swedish patients [2]. Patients risk formation of intracardiac thrombi as a consequence of atrial fibrillation, leading to systemic embolism and ischemic stroke, or hemorrhages due to treatment with anticoagulants. The risk-benefit profile of NOACs has proved superior to warfarin or no treatment [1, $5,6]$. However, multimorbidity and polypharmacy add to the risks requiring attention in the treatment of patients with atrial fibrillation. Hypertension, heart failure, diabetes, stroke, and 
myocardial infarction were the most common comorbidities in this patient group in the pivotal NOAC trials [7]. Furthermore, polypharmacy is frequent, in the ARISTOTLE trial (apixaban) in atrial fibrillation patients, the median number of concomitantly used drugs was 6 , and $76.5 \%$ of the patients fulfilled the definition of polypharmacy ( 5 or more drugs) [8]. Similarly, in the ROCKET-AF trial (rivaroxaban), the median number of concomitant medications was 5 , and $64 \%$ of patients were exposed to polypharmacy [9]. Generally, population-based studies have shown that a higher number of drugs are associated with an increased exposure to potential drug-drug interactions, and associations between polypharmacy and an increased risk of major bleeding or stroke have been implicated in NOAC patients from pivotal studies [10-12].

In general, NOAC drug interactions may arise by two principally different mechanisms. Pharmacodynamic interactions result from concomitant treatment with drugs with individual effects on hemostasis or risk of bleeding. Pharmacokinetic interactions are caused by impact of other drugs on the systemic level of NOACs [1]. P-glycoprotein (P-gp) is a critical drug transporter expressed both in the liver, in the intestinal wall, and in the kidney. This efflux pump promotes lowering of systemic levels of its transporter substrates including all NOACs. In addition, cytochrome P 450 (CYP) 3A4 has an important role in metabolic clearance of apixaban and rivaroxaban in the liver and the intestinal wall [13]. Consequentially, drugs that inhibit or induce P-gp may affect exposure to all NOACs, whereas interactions via CYP3A4 are primarily expected for apixaban and rivaroxaban. Guidelines and recommendations on potential drug interactions with NOACs are primarily based on pharmacokinetic studies, and there are few studies on the overall clinical effects of potential drug interactions with NOACs $[12,14,15]$.

The aim of this study was to evaluate whether concomitant treatment with NOACs and interacting drugs was associated with increased risks of bleeding or thromboembolic events in patients with atrial fibrillation in Swedish outpatient care, compared to the use of NOAC only.

\section{Methods}

\section{Study design and data sources}

This study was a retrospective cohort study, based on data from three national Swedish registers, the Prescribed Drug Register, the Patient Register, and the Cause of Death Register [16]. Patients with atrial fibrillation that had been prescribed any NOAC since the introduction of these drugs in Sweden were included in the cohort. Composite primary endpoints were any severe bleed (gastrointestinal bleeding, hemorrhagic stroke, other intracranial bleeding, other severe bleeding) and ischemic stroke/stroke unspecified/TIA, for potential bleeding and thromboembolism interactions, respectively. Secondary endpoints were selected components of the primary endpoints, gastrointestinal bleeding, intracranial bleeding, and ischemic stroke and the separate endpoint venous thrombosis. The Prescribed Drug Register contains information on all dispensed prescription drugs in Sweden. All drugs prescribed in outpatient health care or drugs taken from drug storage rooms in nursing homes are included in the register, but not over the counter (OTC) drugs or drugs used in hospital inpatient care. Consequentially, outpatient prescription drug use in the country is very well covered [17]. Data retrieved from the Prescribed Drug Register included the code according to the Anatomical Therapeutic Chemical (ATC) classification system of the drug, dispensation date, and the size of the dispensed prescription in defined daily doses (DDDs) [18]. The Patient Register contains information on all healthcare visits in specialized outpatient and inpatient care. Information on diagnosis according to the International Classification of Diseases (ICD), procedure codes, and dates for diagnosis are available for each care event [19]. The Cause of Death Register contains information on the time of all deaths that occur in Sweden, with ICD diagnoses specifying the cause of death and contributing causes. The above registers are maintained by the National Board of Health and Welfare and data can be linked between registers using the Swedish personal identity number, unique to each individual.

\section{Study cohort}

For patients that had been prescribed any NOAC (apixaban, rivaroxaban, dabigatran or edoxaban) between 2008 and 2017, data on all prescribed drugs were retrieved from the Prescribed Drug Register between 2007 and 2017. In addition, data from the Patient Register between 1998 and 2017, and the Cause of Death Register between 2008 and 2017, on diagnoses and procedure codes (Online Resource, Suppl. Table 1), and time of deaths, were retrieved for these patients. The first NOAC was introduced in Sweden in 2008, and 2017 was the last year for which data were available in all registers. Patients with atrial fibrillation and a new initiation of NOAC treatment were included in the study. Indications for NOAC treatment in Sweden include atrial fibrillation, deep venous thrombosis and pulmonary embolism, and postoperative prophylaxis following hip or knee replacement. The Patient Register does not fully cover primary health care, where some patients receive atrial fibrillation diagnoses. In contrast, diagnoses of other indications for NOAC treatment are given mainly in specialized health care. To include patients with presumed atrial fibrillation in the study, patients with diagnosis of any of the other indications within 60 days of initiated NOAC treatment were excluded. Furthermore, patients with mechanical valve or mitral stenosis were excluded. A washout period of 90 days 
before NOAC treatment was implemented for vitamin $\mathrm{K}$ receptor antagonists and previous NOAC treatment.

\section{Definitions of exposure}

Treatment periods for NOAC and interacting drugs were identified through linkage of drug dispensations. Drug treatment was identified based on the ATC-code/s of each substance (Online Resource, Suppl. Table 2). Linked treatment periods were estimated based on the number of DDDs of consecutive dispensations. A dispensation that occurred within the time frame defined by $2.5 \mathrm{x}$ the number of DDDs of the previous dispensation, but less than 100 days after the previous dispensation, was considered a linked continuous treatment. Since the DDD does not always account for the actual prescribed dose, multiplying with 2.5 allowed for a halved dosing regimen to be linked. However, a limit of 100 days prevented overestimation of linkage between dispensations. This limit was set based on the circumstance that patients on long-term treatment are dispensed drugs every third to fourth month, a consequence of the Swedish reimbursement model.

Drugs that potentially interact with NOACs were identified in the Janusmed interactions database [20] and in guidelines from the European Heart Rhythm Association (EHRA) [1]. Clinically relevant interactions defined as contraindicated, or where dose adjustment is recommended (class D or C, respectively, in Janusmed interactions) in any of these two sources, were identified. Potentially interacting drugs were classified according to the mechanism of interaction with each NOAC, into drugs with pharmacodynamic effect on bleeding, CYP3A4 and/or P-gp inhibitors, or CYP3A4 and/or P-gp inducers (Online Resource, Suppl. Table 3). Additionally, drugs that were listed as having a pharmacodynamic effect on bleeding with any NOAC were included for all NOACs in the study. The first episode with a new initiation of NOAC treatment for each patient was included in the analysis. For patients exposed to more than one NOAC, the first treatment episode for each NOAC was included. Potentially interacting concomitant drug treatment initiated before or at the time of initiation of the NOAC defined exposure to a potential interaction.

Descriptive data is presented for baseline variables with proportions or mean values and standard deviations. For all descriptive baseline variables and baseline covariables used in the analyses, three separate time frames were defined to identify ICD diagnoses before the index date. Consequentially, diagnoses within 10 years, 5 years, or 6 months before index date were included depending on clinical relevance (Online Resource, Suppl. Table 1). Patients were followed up for 6 months after initiation of NOAC treatment. Censoring occurred at the end of exposure to either NOAC or the interacting combination or death. Migration was assumed to result in the end of exposure based on the Swedish register data.

\section{Statistical analyses}

Cox-regression survival analyses were performed for all endpoints. Outcome events were defined as receiving either a primary or secondary diagnosis for the outcome in the Patient Register or the Cause of Death register during the time of exposure (Online Resource, Suppl. Table 1). The hazard ratio for bleeding events or thromboembolic events was analyzed for apixaban, rivaroxaban, and dabigatran with or without interacting drugs presumed to potentiate the risk according to the Janusmed interactions database or EHRA guidelines. Patients exposed to edoxaban were too few in the dataset to allow analysis. All analyses were adjusted for the mechanistic interaction groups not currently analyzed. Analyses of risk of bleeding was adjusted for selected components of HAS-BLED [21, 22] (hypertension, renal disease, liver disease, ischemic stroke/stroke unspecified/TIA, any severe bleed, anemia, age category, and alcohol abuse). PK-INR was not available. Additionally, the use of platelet aggregation inhibitors was included in the mechanistic group with pharmacodynamic effect. The analyses of risk of thromboembolism were adjusted for the components of $\mathrm{CHA}_{2} \mathrm{DS}_{2}$-VASc (heart failure, hypertension, diabetes, ischemic stroke/TIA/ arterial embolus/stroke unspecified, vascular disease, age category, and sex) $[22,23]$. Crude outcome free survival probabilities of the primary outcomes were presented in Kaplan-Meier curves.

All variables were categorical in the analyses. To evaluate the proportional hazards assumption, we analyzed Schoenfeld residuals and performed exploratory Cox-regression analyses with covariate-time interactions. In addition, analyses to evaluate statistical interaction between covariates were conducted. We retrieved data on all patients fulfilling inclusion criteria from the Prescribed Drug Register. However, power analyses indicated $83-89 \%$ probability of successfully identifying a hazard ratio (HR) of 2 with 1000 patients exposed to an interaction, depending on the NOAC analyzed.

All statistical analyses and managing of the datasets were performed using R version 3.6.1 [24]. Statistical significance was defined at the 5\% level (two-sided). HRs are presented with $95 \%$ confidence intervals (CI).

\section{Results}

Among outpatients dispensed NOACs in Sweden during the study period, from 2008 to $2017,244,597$ patients with presumed atrial fibrillation had new episodes of NOAC treatment (Fig. 1). The percentages of patients with apixaban, rivaroxaban, and dabigatran were 61,24 , and $15 \%$ (the percentage of patients with edoxaban was less than $1 \%$ ) (Table 1). Potential interactions with pharmacodynamic effect 
Fig. 1 Study inclusion flow chart of 244,597 patients with newly initiated NOAC treatment during 2008-2017 in Swedish outpatient care. Excluded groups of patients are shown in the order of exclusion from the dataset

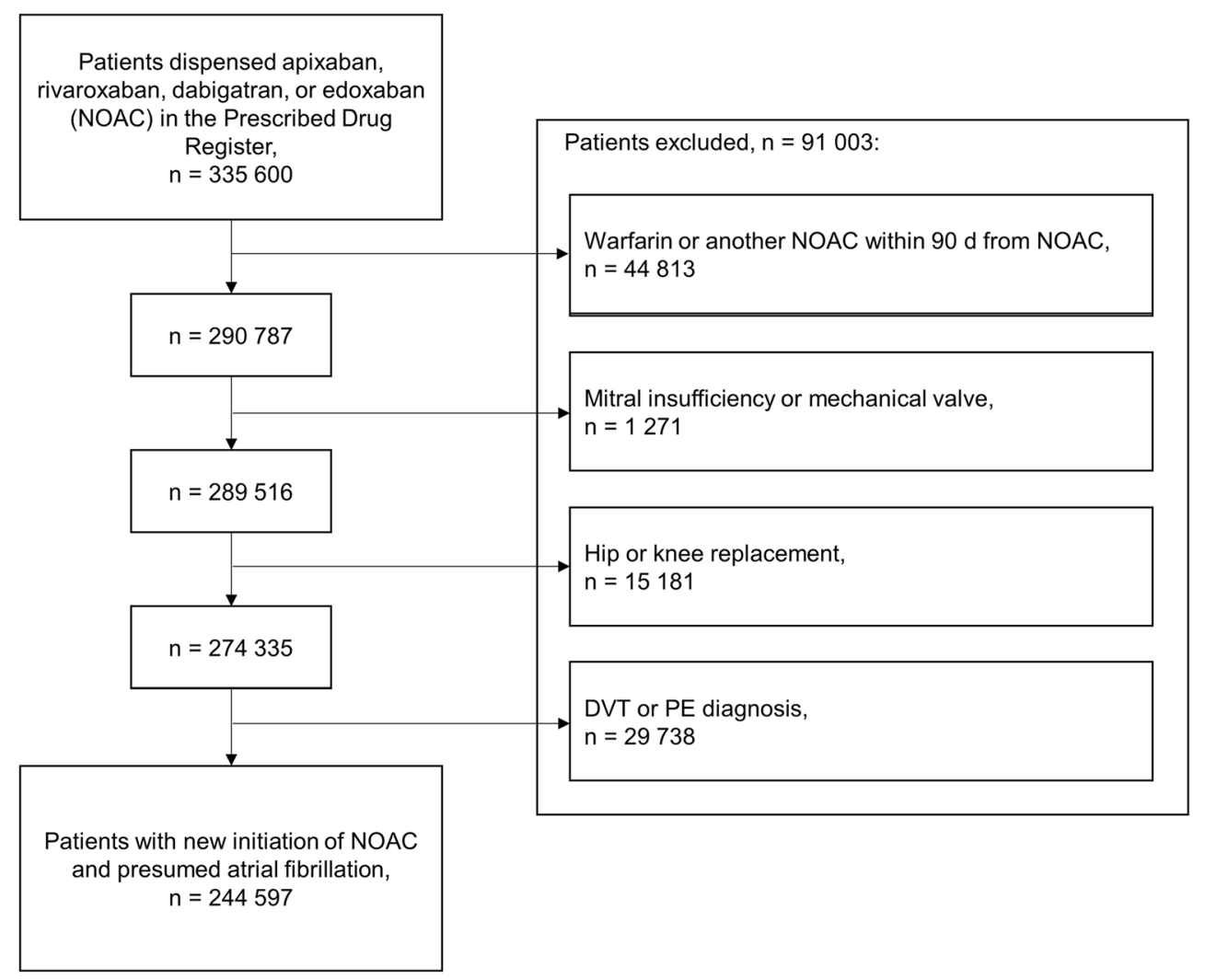

on bleeding were found in $48 \%$ of the patients, whereas inhibitors and inducers of CYP3A4 and/or P-gp were less common, 4 and $1 \%$, respectively. More than half of the patients exposed to inhibitors or inducers of CYP3A4 and/or P-gp also had drugs with pharmacodynamic effect on bleeding, whereas only a few percent of patients with pharmacodynamic interactions had drugs from the pharmacokinetic groups. The distribution of age and sex was similar between patients without potential interactions and patients with the respective interaction groups. Patients without potential interactions had less cardiovascular morbidity, the mean $\mathrm{CHA}_{2} \mathrm{DS}_{2}$-VASc score was lower, and previous bleeding events were less common at baseline. Additionally, other comorbidities, e.g. cancer, diabetes, and renal disease, were more common among patients exposed to potential interactions.

Acetylsalicylic acid (ASA) dominated among drugs with a pharmacodynamic effect on bleeding (Table 2, Online Resource, Suppl. Table 4). Furthermore, clopidogrel and non-steroidal anti-inflammatory drugs (NSAIDs) were relatively common in combination with all NOACs. Drugs that interact pharmacokinetically were uncommon in combination with NOACs, generally less than $1 \%$ of patients for each NOAC.

In the adjusted Cox-regression analyses, pharmacodynamic and pharmacokinetic interactions with potential effect on the risk of bleeding were associated with an increased risk of any severe bleed. The association with drugs with pharmacodynamic effect appeared to be more pronounced for rivaroxaban (HR 1.7; 1.49-1.92), and apixaban (HR $1.47 ; 1.33-1.63$ ) than for dabigatran (HR 1.26; 1.05-1.52) (Fig. 2 and Fig. 5). Patients treated with apixaban that were exposed to CYP3A4 and/or P-gp inhibitors also had an increased risk of any severe bleed (HR 1.23; 1.01-1.5), whereas a significant effect for patients treated with rivaroxaban or dabigatran and these interacting drugs could not be established (rivaroxaban, HR 1.24; 0.94-1.65, and dabigatran HR 0.84; 0.48-1.45) (Fig. 3).

Few patients had inducers of CYP3A4 and/or P-gp in the dataset (Online Resource, Suppl. Table 5). Therefore, estimates of the risk of the composite primary endpoint ischemic stroke/TIA/stroke unspecified associated with this drug group could not be established in the adjusted Cox-regression analyses. Notably however, in this dataset the upper limit of the confidence interval of the endpoint did not exceed 1.57 for any of the NOACs analyzed (Fig. 4).

Among the secondary endpoints, the association of drugs that have a pharmacodynamic effect on bleeding and CYP3A4 and/ or P-gp-inhibitors were associated with an increased risk of gastrointestinal bleeding for apixaban (HR 1.51; 1.28-1.78, and HR $1.44 ; 1.06-1.95)$ and rivaroxaban (HR 1.68; 1.37-2.05, and HR 1.54; 1.02-2.32) (Fig. 2 and Fig. 3). The combined use of apixaban and inducers of CYP3A4 and/or P-gp was associated with an increased risk of venous thromboembolism (HR 1.58; 1.0-2.5) (Fig. 4 and Fig 5). 
Table 1 Baseline characteristics for 244,597 patients with newly initiated NOAC treatment during 2008-2017 in Swedish outpatient care. Values are given as $n(\%)$ or mean ( $\pm \mathrm{SD})$

\begin{tabular}{lllll} 
NOAC & No interaction & Pharmacodynamic effect & CYP3A4 and/or & CYP3A4 and/or \\
$n=244,597$ & $n=122,476$ & $n=116,499$ & $\begin{array}{l}\text { P-gp inhibitor } \\
n=8927\end{array}$ & $\begin{array}{l}\text { P-gp inducer } \\
n=3314\end{array}$ \\
& & & & \\
\hline
\end{tabular}

\begin{tabular}{|c|c|c|c|c|c|}
\hline \multicolumn{6}{|l|}{ NOAC } \\
\hline Apixaban (\%) & $148,108(61)$ & $74,808(61)$ & $69,564(60)$ & $5841(65)$ & $2136(64)$ \\
\hline Rivaroxaban (\%) & $59,276(24)$ & $29,505(24)$ & $28,597(25)$ & $1894(21)$ & $783(24)$ \\
\hline Dabigatran $(\%)$ & $35,617(15)$ & $17,524(14)$ & $17,475(15)$ & $984(11)$ & $375(11)$ \\
\hline Edoxaban $(\%)$ & $1596(<1)$ & $639(<1)$ & $863(<1)$ & $208(2)$ & $20(<1)$ \\
\hline \multicolumn{6}{|l|}{ Interacting drug groups } \\
\hline Pharmacodynamic effect (\%) & $116,499(48)$ & $0(0)$ & $116,499(100)$ & $4596(51)$ & $1974(60)$ \\
\hline CYP3A4 and/or P-gp inhibitor (\%) & $8927(4)$ & $0(0)$ & $4596(4)$ & $8927(100)$ & $126(4)$ \\
\hline CYP3A4 and/or P-gp inducer (\%) & $3314(1)$ & $0(0)$ & $1974(2)$ & $126(1)$ & $3314(100)$ \\
\hline \multicolumn{6}{|l|}{ Age and sex } \\
\hline Age, mean (SD) & $72.1(12.9)$ & $71.1(13.3)$ & $73.2(12.4)$ & $72.4(11.4)$ & $71.9(12.3)$ \\
\hline Age $<65$ years $(\%)$ & $56,057(23)$ & $30,948(25)$ & $23,666(20)$ & $1938(22)$ & $753(23)$ \\
\hline Age $65-74$ years $(\%)$ & $76,467(31)$ & $39,263(32)$ & $35,402(30)$ & $2959(33)$ & $1040(31)$ \\
\hline Age $75-79$ years $(\%)$ & $39,560(16)$ & $18,948(15)$ & $19,678(17)$ & $1570(18)$ & $595(18)$ \\
\hline Age $80+$ years $(\%)$ & $72,513(30)$ & $33,317(27)$ & $37,753(32)$ & $2460(28)$ & $926(28)$ \\
\hline Female $(\%)$ & $114,740(47)$ & $56,811(46)$ & $55,624(48)$ & $3582(40)$ & $1490(45)$ \\
\hline \multicolumn{6}{|l|}{ Cardiovascular morbidity } \\
\hline CHA2DS2VASc, mean (SD) & $2.9(1.9)$ & $2.6(1.8)$ & $3.4(2)$ & $3.1(1.8)$ & $3.5(2)$ \\
\hline Ischemic stroke/stroke unspecified/TIA (\%) & $35,049(14)$ & $11,472(9)$ & $22,859(20)$ & $1140(13)$ & $1085(33)$ \\
\hline Ischemic stroke $(\%)$ & $23,182(9)$ & $7688(6)$ & $14,997(13)$ & $738(8)$ & $865(26)$ \\
\hline Venous thrombosis $(\%)$ & $21,066(9)$ & $9907(8)$ & $10,689(9)$ & $775(9)$ & $488(15)$ \\
\hline Vascular disease $(\%)$ & $52,831(22)$ & $13,805(11)$ & $37,988(33)$ & $2795(31)$ & $830(25)$ \\
\hline Any severe bleed (\%) & $21,045(9)$ & $9015(7)$ & $11,411(10)$ & $1013(11)$ & $510(15)$ \\
\hline Gastrointestinal bleeding (\%) & $7435(3)$ & $3148(3)$ & $4087(4)$ & $352(4)$ & $148(4)$ \\
\hline Hemorrhagic stroke (\%) & $1335(<1)$ & $565(<1)$ & $716(<1)$ & $39(<1)$ & $105(3)$ \\
\hline Heart failure (\%) & $33,619(14)$ & $14,095(12)$ & $18,558(16)$ & $1718(19)$ & $558(17)$ \\
\hline Hypertension (\%) & $113,875(47)$ & $47,977(39)$ & $63,059(54)$ & $5132(57)$ & $1810(55)$ \\
\hline \multicolumn{6}{|l|}{ Other comorbidity } \\
\hline Cancer $(\%)$ & $38,589(16)$ & $16,505(13)$ & $20,372(17)$ & $3024(34)$ & $952(29)$ \\
\hline Anemia $(\%)$ & $4956(2)$ & $1930(2)$ & $2881(2)$ & $253(3)$ & $138(4)$ \\
\hline COPD/emphysema (\%) & $14,138(6)$ & $5877(5)$ & $7852(7)$ & $739(8)$ & $249(8)$ \\
\hline Diabetes $(\%)$ & $32,235(13)$ & $12,285(10)$ & $19,249(17)$ & $1405(16)$ & $532(16)$ \\
\hline Liver disease (\%) & $2521(1)$ & $1175(<1)$ & $1263(1)$ & $141(2)$ & $65(2)$ \\
\hline Renal disease $(\%)$ & $7958(3)$ & $3028(2)$ & $4630(4)$ & $574(6)$ & $175(5)$ \\
\hline Dementia $(\%)$ & $5411(2)$ & $1993(2)$ & $3310(3)$ & $139(2)$ & $176(5)$ \\
\hline Obesity (\%) & $2200(<1)$ & $892(<1)$ & $1246(1)$ & $130(1)$ & $42(1)$ \\
\hline Alcohol abuse $(\%)$ & $1536(<1)$ & $723(<1)$ & $763(<1)$ & $55(<1)$ & $57(2)$ \\
\hline Frequent falls (> 2 registrations) (\%) & $3271(1)$ & $1572(1)$ & $1630(1)$ & $116(1)$ & $72(2)$ \\
\hline
\end{tabular}

Comorbidities indicate current or relevant previous diagnoses at baseline

The proportional hazards assumption and potential interaction between covariables were evaluated. For the analysis of any severe bleed in patients with apixaban and inhibitors of CYP3A4 and/or P-gp, a significant effect of time on the HR was detected. The analysis indicated that the effect may be somewhat larger in the later part of the study period. No statistical interactions were detected between covariables in evaluations preformed for analyses of primary endpoints in the apixaban datasets. 
Table 2 Potential NOAC drug interactions with drugs with a frequency of $\geq 50$ for at least one NOAC at index, in 244,597 patients with newly initiated NOAC treatment during 2008-2017 in Swedish outpatient care. Values are given as $n(\%)$

\begin{tabular}{|c|c|c|c|c|}
\hline & $\begin{array}{l}\text { Apixaban } \\
n=148,108\end{array}$ & $\begin{array}{l}\text { Rivaroxaban } \\
n=59,276\end{array}$ & $\begin{array}{l}\text { Dabigatran } \\
n=35,617\end{array}$ & $\begin{array}{l}\text { Edoxaban } \\
n=1596\end{array}$ \\
\hline \multicolumn{5}{|l|}{ Cardiovascular drugs } \\
\hline Amiodarone & $1368(0.92)$ & $341(0.58)$ & $207(0.58)$ & $38(2.38)$ \\
\hline Diltiazem & $528(0.36)$ & $259(0.44)$ & $195(0.55)$ & $9(0.56)$ \\
\hline Dronedarone & $1135(0.77)$ & $168(0.28)$ & $201(0.56)$ & $129(8.08)$ \\
\hline Verapamil & $962(0.65)$ & $416(0.7)$ & $250(0.7)$ & $16(1)$ \\
\hline \multicolumn{5}{|l|}{ Antiepileptics } \\
\hline Carbamazepine & $525(0.35)$ & $159(0.27)$ & $113(0.32)$ & $19(1.19)$ \\
\hline Levetiracetam & $651(0.44)$ & $248(0.42)$ & $157(0.44)$ & $8(0.5)$ \\
\hline Phenytoin & $118(0.08)$ & $47(0.08)$ & $26(0.07)$ & $4(0.25)$ \\
\hline Valproic acid & $365(0.25)$ & $137(0.23)$ & $61(0.17)$ & $3(0.19)$ \\
\hline \multicolumn{5}{|l|}{ Antibiotics } \\
\hline Clarithromycin & $53(0.04)$ & $19(0.03)$ & $7(0.02)$ & $1(0.06)$ \\
\hline Rifampicin & $56(0.04)$ & $24(0.04)$ & $12(0.03)$ & $5(0.31)$ \\
\hline \multicolumn{5}{|l|}{ Antidepressants } \\
\hline Citalopram & $5401(3.65)$ & $1930(3.26)$ & $1095(3.07)$ & $53(3.32)$ \\
\hline Escitalopram & $1523(1.03)$ & $597(1.01)$ & $282(0.79)$ & $17(1.07)$ \\
\hline Fluoxetine & $438(0.3)$ & $208(0.35)$ & $115(0.32)$ & $3(0.19)$ \\
\hline Paroxetine & $473(0.32)$ & $208(0.35)$ & $120(0.34)$ & $7(0.44)$ \\
\hline Sertraline & $3498(2.36)$ & $1392(2.35)$ & $674(1.89)$ & $24(1.5)$ \\
\hline Clomipramine & $180(0.12)$ & $70(0.12)$ & $39(0.11)$ & $5(0.31)$ \\
\hline Duloxetine & $653(0.44)$ & $282(0.48)$ & $172(0.48)$ & $12(0.75)$ \\
\hline Venlafaxine & $1212(0.82)$ & $517(0.87)$ & $264(0.74)$ & $19(1.19)$ \\
\hline \multicolumn{5}{|l|}{ Antimycotics } \\
\hline Fluconazole & $159(0.11)$ & $78(0.13)$ & $21(0.06)$ & $5(0.31)$ \\
\hline \multicolumn{5}{|l|}{ Antineoplastic agents } \\
\hline Enzalutamide & $50(0.03)$ & $7(0.01)$ & $10(0.03)$ & $3(0.19)$ \\
\hline Bicalutamide & $1033(0.7)$ & $352(0.59)$ & $150(0.42)$ & $13(0.81)$ \\
\hline Tamoxifen & $390(0.26)$ & $192(0.32)$ & $87(0.24)$ & $11(0.69)$ \\
\hline \multicolumn{5}{|c|}{ Immunomodulating agents } \\
\hline Ciclosporin & $76(0.05)$ & $14(0.02)$ & $4(0.01)$ & $2(0.13)$ \\
\hline Dexamethasone & $205(0.14)$ & $49(0.08)$ & $5(0.01)$ & $3(0.19)$ \\
\hline Prednisone & $162(0.11)$ & $90(0.15)$ & $19(0.05)$ & $2(0.13)$ \\
\hline Tacrolimus & $174(0.12)$ & $42(0.07)$ & $7(0.02)$ & $5(0.31)$ \\
\hline \multicolumn{5}{|l|}{ Analgesic } \\
\hline Tramadol & $1520(1.03)$ & $1351(2.28)$ & $2428(6.82)$ & $11(0.69)$ \\
\hline \multicolumn{5}{|l|}{ Antithombotic agents } \\
\hline Acetylsalicylic acid & $39,888(26.93)$ & $14,168(23.9)$ & $8541(23.98)$ & $375(23.5)$ \\
\hline Clopidogrel & $7060(4.77)$ & $2380(4.02)$ & $1191(3.34)$ & $69(4.32)$ \\
\hline Dipyridamole & $808(0.55)$ & $211(0.36)$ & $207(0.58)$ & $2(0.13)$ \\
\hline Ticagrelor & $1202(0.81)$ & $365(0.62)$ & $207(0.58)$ & $17(1.07)$ \\
\hline Dalteparin & $3495(2.36)$ & $1912(3.23)$ & $535(1.5)$ & $145(9.09)$ \\
\hline Enoxaparin & $571(0.39)$ & $236(0.4)$ & $116(0.33)$ & $16(1)$ \\
\hline Tinzaparin & $2488(1.68)$ & $1026(1.73)$ & $279(0.78)$ & $86(5.39)$ \\
\hline \multicolumn{5}{|l|}{ NSAIDs } \\
\hline Celecoxib & $1105(0.75)$ & $154(0.26)$ & $134(0.38)$ & $6(0.38)$ \\
\hline Dexibuprofen & $120(0.08)$ & $80(0.13)$ & $63(0.18)$ & $1(0.06)$ \\
\hline Diclofenac & $2038(1.38)$ & $1622(2.74)$ & $1818(5.1)$ & $7(0.44)$ \\
\hline
\end{tabular}


Table 2 (continued)

\begin{tabular}{|c|c|c|c|c|}
\hline & $\begin{array}{l}\text { Apixaban } \\
n=148,108\end{array}$ & $\begin{array}{l}\text { Rivaroxaban } \\
n=59,276\end{array}$ & $\begin{array}{l}\text { Dabigatran } \\
n=35,617\end{array}$ & $\begin{array}{l}\text { Edoxaban } \\
n=1596\end{array}$ \\
\hline Etoricoxib & 4207 (2.84) & $2845(4.8)$ & $782(2.2)$ & $6(0.38)$ \\
\hline Ibuprofen & $1256(0.85)$ & $666(1.12)$ & $559(1.57)$ & $9(0.56)$ \\
\hline Ketoprofen & $781(0.53)$ & $424(0.72)$ & $420(1.18)$ & $4(0.25)$ \\
\hline Meloxicam & $59(0.04)$ & $29(0.05)$ & $30(0.08)$ & $0(0)$ \\
\hline Nabumetone & $242(0.16)$ & $87(0.15)$ & $308(0.86)$ & $1(0.06)$ \\
\hline Naproxen & $3604(2.43)$ & $1990(3.36)$ & $1098(3.08)$ & 19 (1.19) \\
\hline Tenoxicam & $53(0.04)$ & $30(0.05)$ & $26(0.07)$ & $0(0)$ \\
\hline
\end{tabular}

Percentages based on the total number of patients dispensed each NOAC. The full table is available in Online Resource, Suppl. Table 4

\section{Discussion}

A higher risk of bleeding events was found in patients treated with NOACs and drugs with pharmacodynamic effect on bleeding. The effect of was smaller for dabigatran, and a similar pattern could be seen in secondary endpoints. It should be noted though, that confidence intervals overlap and that we did not formally compare different NOACs in the analysis.

A recently published case-control study of British primary care patients with 393 cases and 1494 controls showed increased risks for major bleeding events in patients treated with any NOAC and drugs with potential pharmacodynamic effect on bleeding [15]. Analyses of individual NOACs in combination with drugs that interact pharmacodynamically indicated higher risk for apixaban and dabigatran but was not statistically significant for rivaroxaban. Individual interacting drugs may have different pharmacodynamic effects on bleeding. The varying results may depend on the distribution of drugs within the group. Furthermore, interactions with a third drug may influence the resulting effect on bleeding. For example patients treated with NOAC and ASA could also be given a proton pump inhibitor (PPI). The concomitant treatment with a PPI lowers dabigatran plasma concentration and may influence the resulting bleeding risk more for dabigatran than for apixaban or rivaroxaban [25].

Fig. 2 Adjusted hazard ratios of outcomes related to co-treatment with NOACs and interacting drugs with pharmacodynamic effect, compared with NOACs without the interacting drug group

Any severe bleed
Apixaban
Rivaroxaban
Dabigatran
Gastrointestinal bleeding
Apixaban
Rivaroxaban
Dabigatran
Intracranial bleeding
Apixaban
Rivaroxaban
Dabigatran

ASA was very frequent in our dataset, and the effect of this drug can be expected to impact highly on the results of the analyses (Table 2, suppl. 1). Clopidogrel and NSAIDs, in contrast, were not as frequent. In the British cohort mentioned above, the effect was more pronounced for ASA, and not significant for clopidogrel or NSAIDs [15]. Therefore, further comparisons in our material between ASA, P2Y12 receptor blockers, and NSAIDs will be a priority in future work.

Among patients treated with apixaban and inhibitors of CYP3A4 and/or P-gp, there was a higher risk of any severe bleed compared with apixaban alone (Fig. 3). Estimates for rivaroxaban and dabigatran were not significantly different with or without these drug interactions. However, there was a trend indicating higher risk estimates for apixaban and rivaroxaban than for dabigatran, and a similar pattern could be seen in the secondary endpoint, gastrointestinal bleeding, a component of the primary endpoint. Apixaban and rivaroxaban are substrates of CYP3A4 and P-gp, whereas dabigatran is a substrate of P-gp only [13, 26]. However, this potential difference in bleeding risk between different NOACs with regard to metabolic drug interactions should be interpreted with caution and warrants further exploration.

In comparison, no statistically significant effect on major bleeding was seen for inhibitors of CYP3A4 and/or P-gp in

\section{Pharmacodynamic effect}

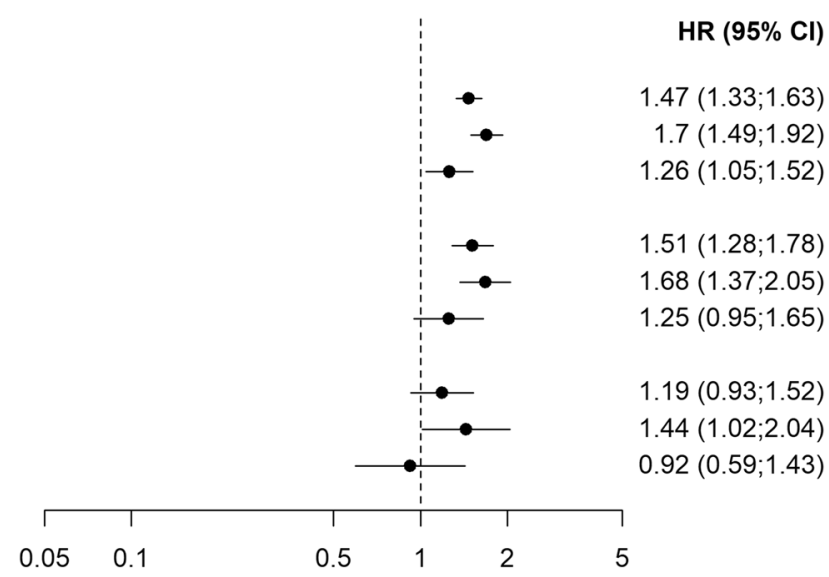


Fig. 3 Adjusted hazard ratios of outcomes related to co-treatment with NOACs and CYP3A4 and/ or P-gp inhibitors, compared with NOACs without the interacting drug group

\section{Inhibitor CYP3A4 and/or P-gp}

Any severe bleed
Apixaban
Rivaroxaban
Dabigatran
Gastrointestinal bleeding
Apixaban
Rivaroxaban
Dabigatran
Intracranial bleeding
Apixaban
Rivaroxaban
Dabigatran

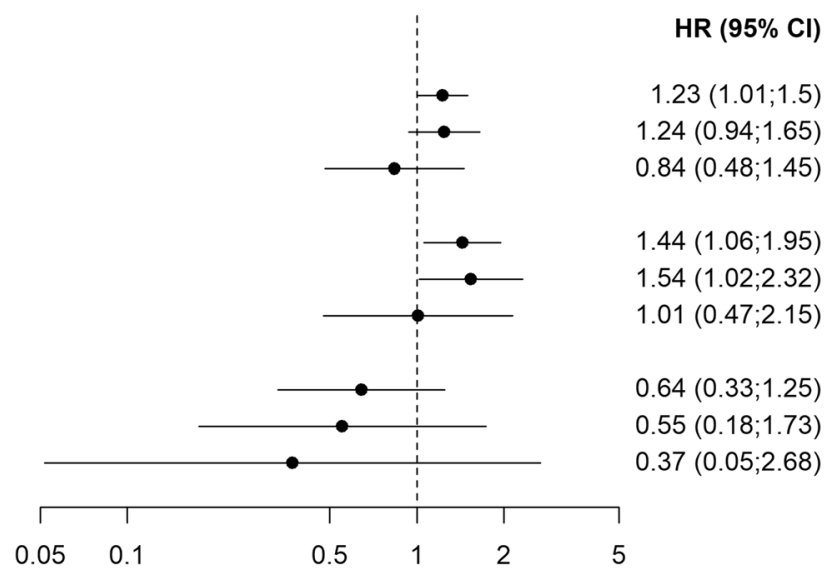

In a meta-analysis based on data from the ARISTOTLE and ROCKET-AF trials, comparisons with warfarin for apixaban and rivaroxaban are presented stratified on interacting drug groups [12]. The risk of major bleeding was higher for rivaroxaban in combination with at least one combined CYP3A4 and P-gp inhibitor compared with warfarin. For aggregated data of apixaban and rivaroxaban, and analyses of each drug, with drugs affecting CYP3A4 and P-gp metabolism, no other significant differences were found in comparison with warfarin. However, for both stroke and major bleeding, estimates of RRs compared with warfarin were higher for patients with drugs affecting metabolism through CYP3A4 and P-gp than for patients without those drugs in the aggregated data and in separate analyses of apixaban and rivaroxaban.

An important strength of this study was the use of a large nationwide cohort with all outpatient drug use from the introduction of NOAC in 2008, until 2017. In addition, drugs that interact with NOACs were defined based on the Janusmed interactions database and in guidelines from EHRA, sources that are used in the clinical setting in Sweden and Europe [1, 17, 30]. Furthermore, the use of NOACs is increasing and
Fig. 4 Adjusted hazard ratios of outcomes related to co-treatment with NOACs and CYP3A4 and/ or P-gp inducers, compared with NOACs without the interacting drug group

\section{Inducer CYP3A4 and/or P-gp}

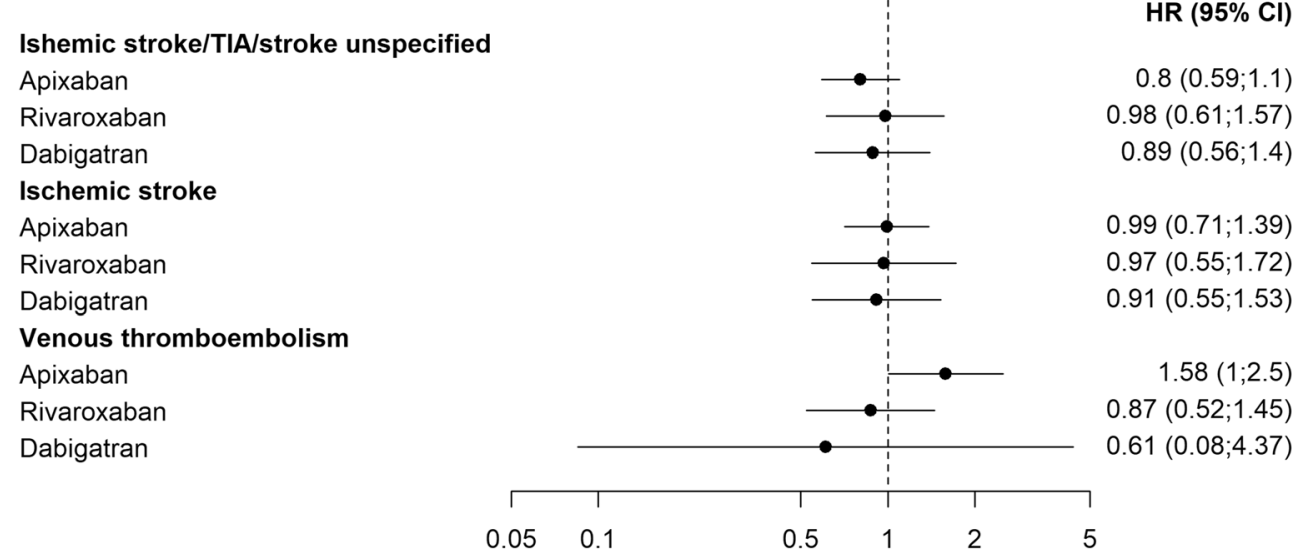




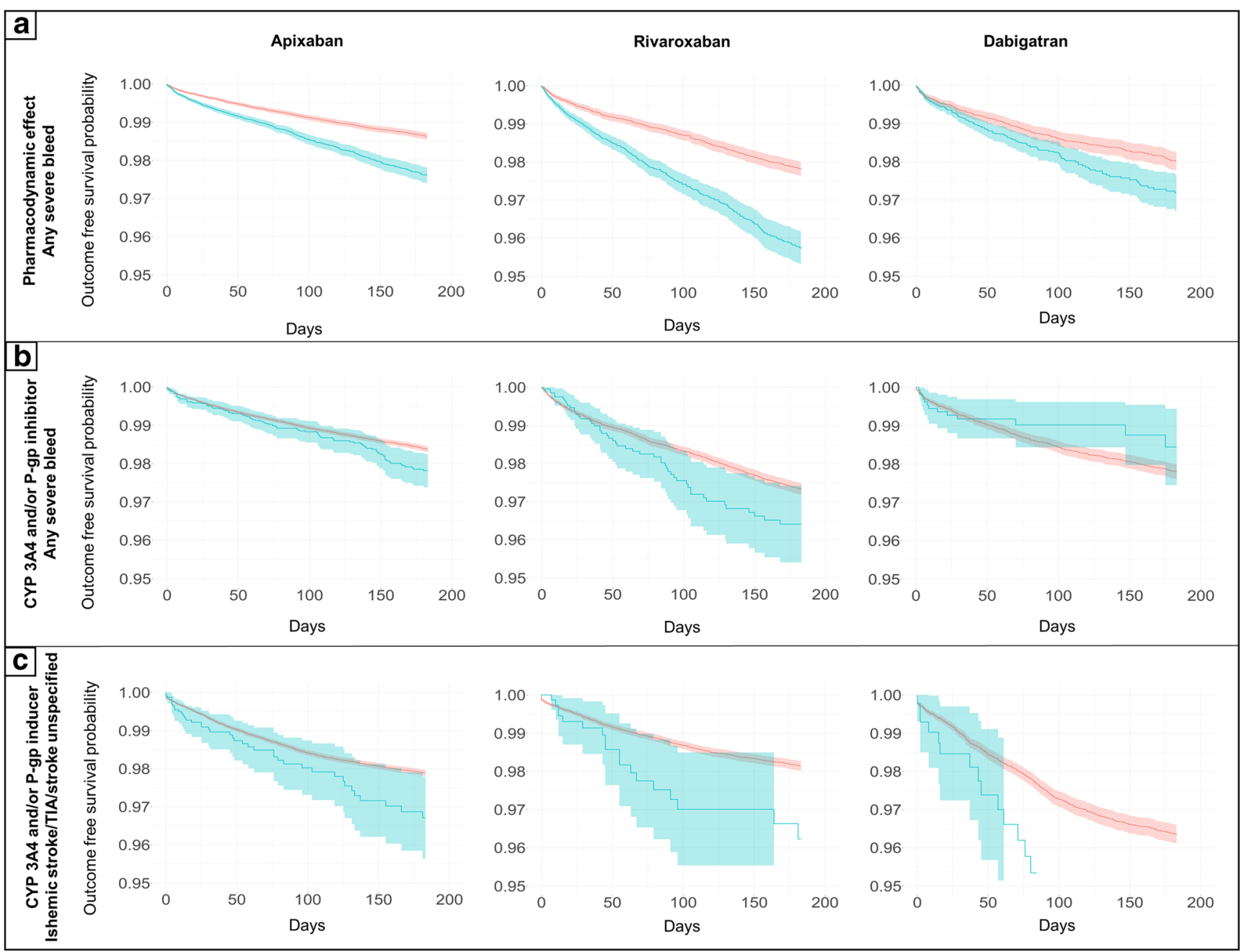

Fig. 5 Unadjusted Kaplan-Meier curves of the effect of interactions with pharmacodynamic effect, CYP 3A4 and/or P-gp inhibitor, and CYP 3A4 and/or P-gp inducer, on the primary composite endpoints any severe bleed or ischemic stroke/TIA/stroke unspecified, respectively. Interacting drug group vs. no exposure to interacting drug group for each NOAC. Blue: Exposed to interaction with pharmacodynamic effect,

evaluation of risk-benefit aspects of treatment is continuously needed $[2,12,14,15]$.

Patients treated with NOAC without any potentially interacting drugs had lower mean $\mathrm{CHA}_{2} \mathrm{DS}_{2}$-VASc, less cardiovascular morbidity, and lower frequencies of other comorbidities (Table 1). Selected components HAS-BLED and $\mathrm{CHA}_{2} \mathrm{DS}_{2^{-}}$ VASc were included as covariables in the analyses, but it is probable that there may be residual confounding that could not be controlled for in this dataset. Data in this study come from large nationwide registers, which is a strength from a power perspective. However, the limited information in the registers on the specific health status of each patient is a limitation. In addition, OTC drugs, e.g., ASA and NSAIDs, are not included in the Prescribed Drug Register, and the use of these drugs may differ in different patient groups. Furthermore, we did not have access to specific dosing information. Therefore, analyses could inhibiting- or inducing effect on CYP3A4 and/or P-gp, respectively. Red: Not exposed to interaction with pharmacodynamic effect, inhibiting- or inducing effect on CYP3A4 and/or P-gp, respectively. Note that the $y$-axis is truncated to $95 \%$. Panel A: Interactions with pharmacodynamic effect. Panel B: Interactions via inhibition of CYP 3A4 and/or P-gp. Panel C: Interactions via induction of CYP 3A4 and/or P-gp

not be controlled for dose adjustment in NOACs or the potentially interacting drugs. Dose adjustment is a way to handle drugdrug interactions clinically and may lead to an exposure and risk profile that is similar to patients not treated with the interacting drug, which in turn may lead to reduced effect estimates in our study [1].

Analyses of the effect of CYP3A4 and/or P-gp inducers on the risk of thromboembolic events failed to show any difference in risk compared with patients without those drugs. Also, in some cases, HRs were lower for the patients exposed to interacting drugs, but with wide confidence intervals allowing for the possibility that an effect of the interaction may exist but could not be estimated. Due to the relatively small number of patients treated with these drugs in the cohort, this was not an unexpected result of the analyses (Online Resource, Suppl. Table 5). 
In this study, we chose to analyze the risk of bleeding or thromboembolism depending on the expected interaction effect. However, an alternative approach could have been to analyze the net clinical risk and to use a composite endpoint with both bleeding and thromboembolism. Such a design would have the benefit of quantifying the general risk but would not have allowed an evaluation of the clinical effect related to the expected mechanism of different drug interactions.

Censoring was performed based on cessation of drug treatment or death. We did not include competing risks in the analyses. The rationale for this was based on the consideration that drug treatment with NOAC may be discontinued or not depending on the clinical situation, and therefore an accurate definition of relevant competing events could not easily be identified.

We performed multiple analyses of different combinations of drugs in this observational study, and therefore multiple testing may be considered an issue. Whether adjustment for multiple testing should be performed in observational studies or not have been discussed, and different opinions exist on the matter [31,32]. We chose not to adjust for multiple testing and the results should be considered exploratory.

\section{Conclusion}

The results of this nationwide study indicate a higher risk of any severe bleed for patients exposed to drugs that interact pharmacodynamically with NOACs. Furthermore, inhibitors of CYP3A4 and/or P-gp increased the risk of bleeding, whereas results for inducers of CYP3A4 and or P-gp were limited due to the small number of exposed patients. In conclusion, the increased risk with co-prescription of NOACs and interacting drugs is important to consider in clinical practice. Furthermore, to evaluate risks in large groups of patients, additional evaluations in observational studies, in particular focusing on less common combinations with potential clinical relevance, are needed as NOAC use increase with time.

Acknowledgements We would like to thank Mia von Euler, Pauline Raaschou, and Diana Rydberg for discussions on methodology. Mia von Euler, Karolina Nowinski, and Staffan Rosenborg were helpful in discussions on aspects of neurology, cardiology, and renal medicine relevant to the study design. We would also like to thank Paul Dickman for discussions on the evaluation of statistical analyses.

Authors' Contribution Concept and study design: JH, EE, and JDL. All authors contributed to discussions on design. Data management JH and JDL. Analyses: JH. All authors contributed to interpretation of the analyses. Manuscript writing, revision, and approval for publication: all authors.

Funding Open access funding provided by Karolinska Institutet. This study was supported by grants from the Swedish Stroke Association and Region Stockholm.

Availability of data and material The data that support the findings of this study are available from the Swedish National Board of Health and
Welfare. Restrictions apply to the availability of these data, which were used under license for this study. Data are available from the authors upon reasonable request and with the permission of the National Board of Health and Welfare.

\section{Compliance with ethical standards}

Conflict of interest The authors declare that they have no conflict of interest.

Ethics approval Ethical approval of the study was obtained from the Regional Ethics Committee in Stockholm, Karolinska Institutet, 2018/207-31. The use of register data from the Prescribed Drug Register, the Patient Register, and the Cause of Death Register was also approved by the Swedish National Board of Health and Welfare. Data were fully anonymized before access was given to the researchers. The Regional Ethics Committee did not require informed consent to be obtained from the individuals included in the study.

\section{Consent for publication Not applicable.}

Code availability Not applicable

Open Access This article is licensed under a Creative Commons Attribution 4.0 International License, which permits use, sharing, adaptation, distribution and reproduction in any medium or format, as long as you give appropriate credit to the original author(s) and the source, provide a link to the Creative Commons licence, and indicate if changes were made. The images or other third party material in this article are included in the article's Creative Commons licence, unless indicated otherwise in a credit line to the material. If material is not included in the article's Creative Commons licence and your intended use is not permitted by statutory regulation or exceeds the permitted use, you will need to obtain permission directly from the copyright holder. To view a copy of this licence, visit http://creativecommons.org/licenses/by/4.0/.

\section{References}

1. Steffel J, Verhamme P, Potpara TS, Albaladejo P, Antz M, Desteghe L, Haeusler KG, Oldgren J, Reinecke H, RoldanSchilling V, Rowell N, Sinnaeve P, Collins R, Camm AJ, Heidbuchel H (2018) The 2018 European heart rhythm association practical guide on the use of non-vitamin $\mathrm{K}$ antagonist oral anticoagulants in patients with atrial fibrillation. Eur Heart J 39(16): 1330-1393. https://doi.org/10.1093/eurheartj/ehy136

2. The National Board of Health and Welfare (2020) Statistikdatabas läkemedel The National Board of Health and Welfare https://www. socialstyrelsen.se/statistik-och-data/statistik/statistikamnen/ lakemedel/. Accessed June 222020

3. Zhu J, Alexander GC, Nazarian S, Segal JB, Wu AW (2018) Trends and variation in Oral anticoagulant choice in patients with atrial fibrillation, 2010-2017. Pharmacotherapy 38(9):907-920. https://doi.org/10.1002/phar.2158

4. Huisman MV, Rothman KJ, Paquette M, Teutsch C, Diener HC, Dubner SJ, Halperin JL, Ma C, Zint K, Elsaesser A, Bartels DB, Lip GY (2015) Antithrombotic treatment patterns in patients with newly diagnosed Nonvalvular atrial fibrillation: the GLORIA-AF registry, phase II. Am J Med 128(12):1306-1313.e1301. https://doi. org/10.1016/j.amjmed.2015.07.013

5. Komen JJ, Forslund T, Mantel-Teeuwisse AK, Klungel OH, von Euler M, Braunschweig F, Wallen H, Hjemdahl P (2019) 
Association of Preceding Antithrombotic Therapy in atrial fibrillation patients with ischemic stroke, intracranial hemorrhage, or gastrointestinal bleed and mortality. Eur Heart J Cardiovasc Pharmacother. https://doi.org/10.1093/ehjcvp/pvz063

6. Ruff CT, Giugliano RP, Braunwald E, Hoffman EB, Deenadayalu N, Ezekowitz MD, Camm AJ, Weitz JI, Lewis BS, Parkhomenko A, Yamashita T, Antman EM (2014) Comparison of the efficacy and safety of new oral anticoagulants with warfarin in patients with atrial fibrillation: a meta-analysis of randomised trials. Lancet 383(9921):955-962. https://doi.org/10.1016/s0140-6736(13) 62343-0

7. Shaikh F, Pasch LB, Newton PJ, Bajorek BV, Ferguson C (2018) Addressing multimorbidity and Polypharmacy in individuals with atrial fibrillation. Curr Cardiol Rep 20(5):32. https://doi.org/10. 1007/s11886-018-0975-x

8. Jaspers Focks J, Brouwer MA, Wojdyla DM, Thomas L, Lopes RD, Washam JB, Lanas F, Xavier D, Husted S, Wallentin L, Alexander JH, Granger CB, Verheugt FW (2016) Polypharmacy and effects of apixaban versus warfarin in patients with atrial fibrillation: post hoc analysis of the ARISTOTLE trial. Bmj 353:i2868. https://doi.org/10.1136/bmj.i2868

9. Piccini JP, Hellkamp AS, Washam JB, Becker RC, Breithardt G, Berkowitz SD, Halperin JL, Hankey GJ, Hacke W, Mahaffey KW, Nessel CC, Singer DE, Fox KA, Patel MR (2016) Polypharmacy and the efficacy and safety of rivaroxaban versus warfarin in the prevention of stroke in patients with Nonvalvular atrial fibrillation. Circulation 133(4):352-360. https://doi.org/10.1161/ circulationaha.115.018544

10. Guthrie B, Makubate B, Hernandez-Santiago V, Dreischulte T (2015) The rising tide of polypharmacy and drug-drug interactions: population database analysis 1995-2010. BMC Med 13:74. https:// doi.org/10.1186/s12916-015-0322-7

11. Holm J, Eiermann B, Eliasson E, Mannheimer B (2014) A limited number of prescribed drugs account for the great majority of drugdrug interactions. Eur J Clin Pharmacol 70(11):1375-1383. https:// doi.org/10.1007/s00228-014-1745-3

12. Harskamp RE, Teichert M, Lucassen WAM, van Weert H, Lopes RD (2019) Impact of polypharmacy and P-glycoprotein- and CYP3A4-modulating drugs on safety and efficacy of oral anticoagulation therapy in patients with atrial fibrillation. Cardiovasc Drugs Ther 33:615-623. https://doi.org/10.1007/ s10557-019-06907-8

13. Heidbuchel H, Verhamme P, Alings M, Antz M, Diener HC, Hacke W, Oldgren J, Sinnaeve P, Camm AJ, Kirchhof P (2015) Updated European heart rhythm association practical guide on the use of non-vitamin $\mathrm{K}$ antagonist anticoagulants in patients with nonvalvular atrial fibrillation. Europace 17(10):1467-1507. https:// doi.org/10.1093/europace/euv309

14. Chang SH, Chou IJ, Yeh YH, Chiou MJ, Wen MS, Kuo CT, See LC, Kuo CF (2017) Association between use of non-vitamin K Oral anticoagulants with and without concurrent medications and risk of major bleeding in nonvalvular atrial fibrillation. Jama 318(13): 1250-1259. https://doi.org/10.1001/jama.2017.13883

15. Zhang Y, Souverein PC, Gardarsdottir H, van den Ham HA, Maitland-van der Zee AH, de Boer A (2020) Risk of major bleeding among users of direct oral anticoagulants combined with interacting drugs: a population-based nested case-control study. Br J Clin Pharmacol 86(6):1150-1164. https://doi.org/10.1111/bcp.14227

16. The National Board of Health and Welfare (2019) Register. The National Board of Health and Welfare. www.socialstyrelsen.se/ statistik-och-data/register/. Accessed June 122020

17. Wettermark B, Hammar N, Fored CM, Leimanis A, Otterblad Olausson P, Bergman U, Persson I, Sundstrom A, Westerholm B, Rosen M (2007) The new Swedish prescribed drug registeropportunities for pharmacoepidemiological research and experience from the first six months. Pharmacoepidemiol Drug Saf 16(7):726-735. https://doi.org/10.1002/pds.1294

18. WHO Collaborating Centre for Drug Statistics Methodology (2019) ATC/DDD Index. www.whocc.no/atc_ddd_index/. Accessed April 092020

19. World Health Organization (2020) Classifications. World Health Organization. www.who.int/classifications/icd/en/. Accessed October 312019

20. Bottiger Y, Laine K, Andersson ML, Korhonen T, Molin B, Ovesjo ML, Tirkkonen T, Rane A, Gustafsson LL, Eiermann B (2009) SFINX-a drug-drug interaction database designed for clinical decision support systems. Eur J Clin Pharmacol 65(6):627-633. https:// doi.org/10.1007/s00228-008-0612-5

21. Pisters R, Lane DA, Nieuwlaat R, de Vos CB, Crijns HJGM, Lip GYH (2010) A novel user-friendly score (HAS-BLED) to assess 1year risk of major bleeding in patients with atrial fibrillation: the euro heart survey. Chest 138(5):1093-1100. https://doi.org/10. 1378/chest.10-0134

22. Camm AJ, Kirchhof P, Lip GY, Schotten U, Savelieva I, Ernst S, Van Gelder IC, Al-Attar N, Hindricks G, Prendergast B, Heidbuchel H, Alfieri O, Angelini A, Atar D, Colonna P, De Caterina R, De Sutter J, Goette A, Gorenek B, Heldal M, Hohloser SH, Kolh P, Le Heuzey JY, Ponikowski P, Rutten FH (2010) Guidelines for the management of atrial fibrillation: the task force for the management of atrial fibrillation of the European Society of Cardiology (ESC). Eur Heart J 31(19):2369-2429. https://doi.org/10.1093/eurheartj/ehq278

23. Lip GYH, Nieuwlaat R, Pisters R, Lane DA, Crijns HJGM (2010) Refining clinical risk stratification for predicting stroke and thromboembolism in atrial fibrillation using a novel risk factor-based approach: the euro heart survey on atrial fibrillation. Chest 137(2):263-272. https://doi.org/10.1378/chest.09-1584

24. R Core Team (2019) R: a language and environment for statistical computing. R Foundation for Statistical Computing. https://www. R-project.org/

25. Bolek T, Samoš M, Stančiaková L, Ivanková J, Škorňová I, Staško J, Galajda P, Kubisz P, Mokáň M (2019) The impact of proton pump inhibition on Dabigatran levels in patients with atrial fibrillation. Am J Ther 26(3):e308-e313. https://doi.org/10.1097/mjt. 0000000000000599

26. Lund M, Petersen TS, Dalhoff KP (2017) Clinical implications of P-glycoprotein modulation in drug-drug interactions. Drugs 77(8): 859-883. https://doi.org/10.1007/s40265-017-0729-x

27. Linnebur SA, Hanlon JT (2018) Drug interactions with non-vitamin K Oral anticoagulants. Jama 319(8):828-829. https://doi.org/10. 1001/jama.2017.20834

28. Chang SH, Wen MS, Kuo CF (2018) Drug interactions with nonvitamin K Oral anticoagulants-reply. Jama 319(8):830-831. https:// doi.org/10.1001/jama.2017.20854

29. Sennesael AL, Henrard S, Spinewine A (2018) Drug interactions with non-vitamin K Oral anticoagulants. Jama 319(8):829. https:// doi.org/10.1001/jama.2017.20846

30. Andersson ML, Böttiger Y, Bastholm-Rahmner P, Ovesjö ML, Vég A, Eiermann B (2015) Evaluation of usage patterns and user perception of the drug-drug interaction database SFINX. Int J Med Inform 84(5):327-333. https://doi.org/10.1016/j.jimedinf.2015.01. 013

31. Bender R, Lange S (2001) Adjusting for multiple testing-when and how? J Clin Epidemiol 54(4):343-349. https://doi.org/10.1016/ s0895-4356(00)00314-0

32. Rothman KJ (1990) No adjustments are needed for multiple comparisons. Epidemiology 1(1):43-46

Publisher's note Springer Nature remains neutral with regard to jurisdictional claims in published maps and institutional affiliations. 